\title{
Ionization mechanisms in jet-dominated Seyferts: A detailed case study
}

\author{
D. J. Rosario ${ }^{1}$, M. Whittle ${ }^{1}$, J. D. Silverman ${ }^{1}$, A. S. Wilson ${ }^{2}$ \\ and C. H. Nelson ${ }^{3}$ \\ ${ }^{1}$ Astronomy Department, University of Virginia, Charlottesville, VA 22903, USA \\ ${ }^{2}$ Astronomy Department, University of Maryland, College Park, MD 20742, USA \\ ${ }^{3}$ Physics and Astronomy, Drake University, Des Moines, IA 50311-4505, USA
}

\begin{abstract}
For the past 10 years there has been an active debate over whether fast shocks play an important role in ionizing emission line regions in Seyfert galaxies. To investigate this claim, we have studied the Seyfert 2 galaxy Mkn 78, using HST UV/optical images and spectroscopy. Since Mkn 78 provides the archetypal jet-driven bipolar velocity field, if shocks are important anywhere they should be important in this object. Having mapped the emission line fluxes and velocity field, we first compare the ionization conditions to standard photoionization and shock models. We find coherent variations of ionization consistent with photoionization model sequences which combine optically thick and thin gas, but are inconsistent with either autoionizing shock models or photoionization models of just optically thick gas. Furthermore, we find absolutely no link between the ionization of the gas and its kinematic state, while we do find a simple decline of ionization degree with radius. We feel this object provides the strongest case to date against the importance of shock related ionization in Seyferts.
\end{abstract}

\section{Introduction}

Ionization studies of Seyferts have a long history. Early work led to the establishment of nuclear photoionization as the favored NLR ionizing mechanism. But in the past decade or so, standard models have been called into question because, among other reasons, they strongly underestimate the strengths of many of the weaker high-ionization and highexcitation lines (see Binette, Wilson \& Storchi-Bergmann 1996; Robinson et al. 2000 for a more complete discussion). This led to the development of alternative models, as well as refinements to standard nuclear photoionization. In particular, photoionizing shocks, driven by AGN jets and outflows, have emerged as a viable ionizing source, following work by Viegas-Aldrovandi \& Contini (1989) and Dopita \& Sutherland (1996).

We try to resolve this debate by taking the following approach: we choose a Seyfert with strong, NLR-wide jet-gas interactions. If shocks are important in providing the ionizing power in Seyferts, we should expect to see unambiguous signs of their presence in this object's spectrum. If not, current refinements to nuclear photoionization can be tested.

\section{Mkn 78 : A Jet-Gas Interaction Archetype}

The Seyfert 2 Mkn 78 was selected as a target because it lies well off the virial correlation for Seyferts (Whittle 1992), indicating the presence of widespread non-gravitational motions in the ionized gas. This makes it one of the best candidates for a strong radio jet/ISM interaction among the sample of nearby Seyferts. Whittle \& Wilson (2004) discuss the structural aspects of the interaction in detail. 
We use an extensive dataset consisting of HST-STIS longslit spectra from four slits sampling all the major emission line features in the NLR at high spatial resolution $(\sim 0.05$ arcsec). Our spectra give us almost complete FUV and optical wavelength coverage, allowing the measurement of many lines of different ionization state and excitation level. In addition, medium resolution $(\sim 30 \mathrm{~km} / \mathrm{s})$ spectra allow us to accurately estimate the kinematics of the line emitting gas.

\section{Ionization Mechanisms}

We consider three types of ionization models to compare to the observations.

\subsection{Standard Nuclear Photoionization}

Early photoionization models (e.g., Davidson \& Netzer 1979) invoked a population of Lyman thick (ionization-bounded or IB) clouds illuminated by a power-law AGN ionizing continuum (of the form $F_{\nu} \propto \nu^{\alpha}$ ). Using CLOUDY (Version 94.0, Ferland 1996), we ran a set of constant density $\alpha=-1.0$ and $\alpha=-1.4$ models, with sequences in the ionization parameter $U=n_{i} / n_{e}=10^{-1}-10^{-3}$. In all cases, the $\alpha=-1.4$ model was the better match to the data.

\subsection{Multi-Component Nuclear Photoionization : the $\boldsymbol{A}_{\boldsymbol{m} / \boldsymbol{i}}$ Sequence}

The $U$ models can be generalized by the introdution of matter-bounded or MB clouds that are optically thin to the Lyman continuum (Binette et al. 1996). By varying $A_{m / i}$, the relative contribution of the two components to the spectrum, the range of observed emission line ratios can be reproduced for average Seyfert NLRs. Mkn 78, however, has unusually weak high-ionization lines and so we used CLOUDY to generate an $A_{m / i}$ sequence with the ionization parameter of the MB component reduced by a factor of 4 compared to the Binette et al. (1996) value, which allowed us to adequately match [NeV] and other high-ionization lines.

\subsection{Shock models}

We use the Dopita \& Sutherland (1996) shock models, in which the hot postshock gas generates a photoionized quiescent "precursor" region. In this way, shock models are inherently two-component in nature, but with a self-consistent prescription for the relative line contribution from postshock and precursor material. We use models with shock velocity $V_{s h}=200-500 \mathrm{~km} / \mathrm{s}$ and magnetic parameter $B / \sqrt{n}=0-2 \mu G \mathrm{~cm}^{\frac{3}{2}}$. We have also included a more recent sequence of equipartition magnetic shocks from Allen (2004), with $V_{s h}=200-1000 \mathrm{~km} / \mathrm{s}$.

\section{Methods of Analysis}

We employ a series of tests to search for signs of shock excited gas and/or check the consistency of the AGN photoionization paradigm.

\subsection{Line-ratio vs. Line-ratio Diagrams}

Here, we use the well-known method from Baldwin, Phillips \& Terlevich (1981) of plotting sets of line-ratios vs. other line-ratios and comparing the data to the predictions of models. We divide these diagrams into three basic types:

1. Excitation diagrams, such as $[\mathrm{OIII}] \lambda 5007 /[\mathrm{OII}] \lambda 3727$ vs. $[\mathrm{OIII}] / \mathrm{H} \beta[\mathrm{Fig} .1(a)]$. As expected, the data points lie in the intersection space of all the models, with implied $U \sim 10^{-2}-10^{-3}, A_{m / i} \sim 0.2-10.0$ and $V_{s h} \sim 300-500 \mathrm{~km} / \mathrm{s}$. However, trends in the data marginally support nuclear photoionization over shocks. 

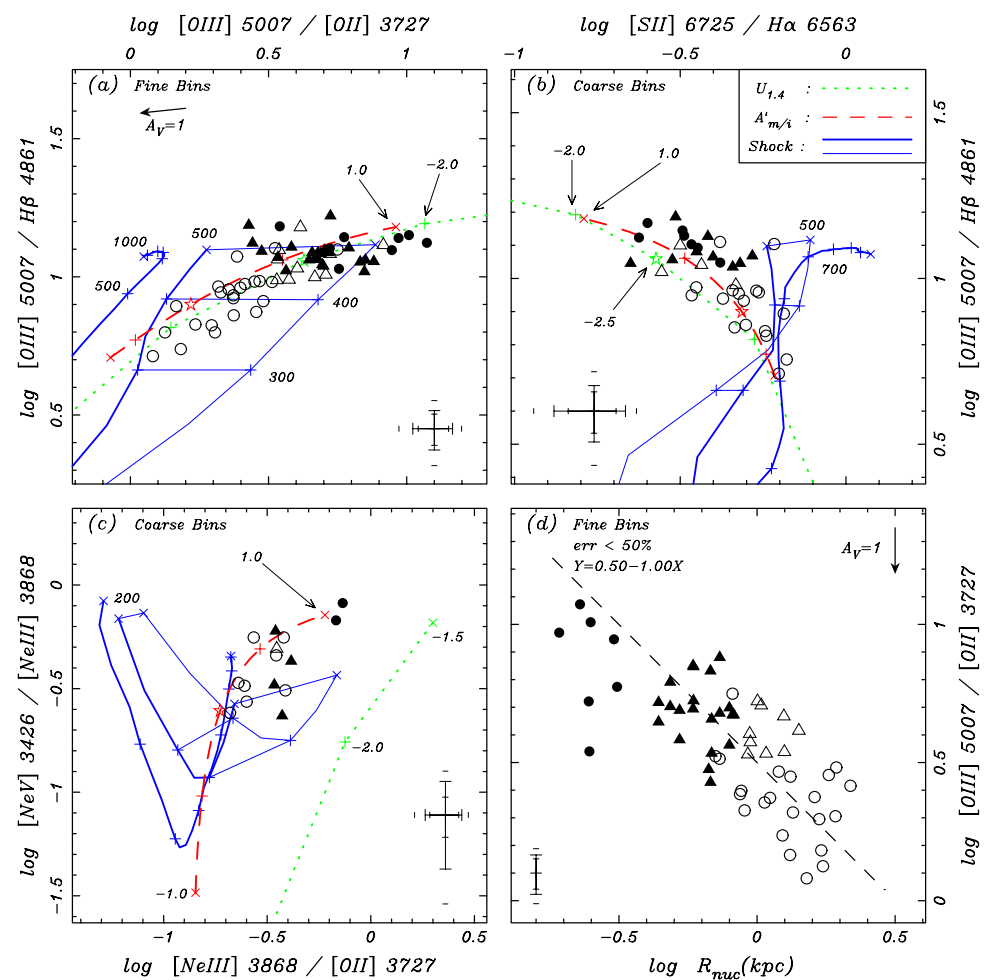

Figure 1. (a,b,c) Emission line-ratio plots. Triangles/Circles : East/West NLR. Filled/Open : Inner/Outer NLR. Three model sequences: dotted line - PL AGN photoionization, $\alpha=-1.4$, dashed line $-A_{m / i}$, solid line - shocks, thick/thin line: magnetic/non-magnetic shocks. All data corrected for extinction. Solid error bars are median $2 \sigma$, with smaller ticks for the 10 and 90 percentile range. On the model loci, crosses are at the bottom of the sequence $(\log U=-3.5$, $\left.\log A_{m / i}=-1.0, V_{s h}=200 \mathrm{~km} / \mathrm{s}\right)$ and at the top of the sequence $\left(\log U=-1.5, \log A_{m / i}=1.0\right.$, $V_{s h}=500$ or $1000 \mathrm{~km} / \mathrm{s}$ ), plus signs are uniform steps in $\log U$ or $A_{m / i}=0.5$ and $V_{s h}=100$ $\mathrm{km} / \mathrm{s}$. (d) Excitation vs. radius. The dashed line with a slope of -1 is not a fit. See Whittle et al. (2004)a for explanation of the binning scheme.

2. Shock discriminators, such as $[\mathrm{SII}] \lambda 6720 / \mathrm{H} \alpha$ vs. $[\mathrm{OIII}] / \mathrm{H} \beta[$ Fig. $1(b)]$. The shock sequences lie almost perpendicular to the photoionization sequences and the trends in the data clearly follow the $U$ and $A_{m / i}$ model loci.

3. U discriminators, which use line-ratios known to be troublesome for standard photoionization. Fig. $1(c)$ shows $[\mathrm{NeIII}] \lambda 3868 /[\mathrm{OII}]$ vs. $[\mathrm{NeV}] /[\mathrm{NeIII}]$. The U models predict far weaker $[\mathrm{NeV}]$ than is measured, even though this line is already unusually weak in Mkn 78.

We conclude that nuclear photoionization probably dominates the line emission processes in the NLR of Mkn 78, with a mixture of optically thin and optically thick gas needed to explain the range of excitation.

\subsection{Line-ratios vs. Other Quantities}

We can measure a host of physical and dynamical properties from our spectra, which we then compare to model predictions. For example, the Dopita \& Sutherland (1996) shock models predict strong correlations between shock velocity and excitation tracing lineratios like $[\mathrm{OIII}] / \mathrm{H} \beta$. On the other hand, the data seems to show absolutely no correlation between excitation and either bulk line velocity or FWHM. There does appear to be, 
however, a strong, significant drop in ionization state with distance from the nucleus, with $[\mathrm{OIII}] /[\mathrm{OII}] \propto r^{-1}[$ Fig. $1(d)]$. A proper interpretation of this trend is, nevertheless, quite complicated. Here, we can only note that the radial trend is evidence for a nuclear ionizing field in the NLR.

\subsection{Profile Comparisons}

A hallmark of two-component models is that the line spectrum of each component is radically different. In the case of shocks, most of the flux in high ionization lines is produced in the kinematically quiescent precursor, while the strongly disturbed postshock cooling region generates a low-ionization spectrum. Thus, it is reasonable to expect a shockexcited spectrum to show significantly different line kinematics between low-ionization lines, like [NII] $\lambda 6584$ and high ionization lines like [OIII]. This is applicable to the MB/IB scenario as well and can be used to set constraints on the level of co-spatiality of the two components.

To look for profile differences, we compared the [OIII] line profile to the profiles of a number of lines of different ionization species, after correcting for the wavelength shift and instrumental broadening. Only very minor differences were found, even in areas with strong signs of jet-gas interaction. We conclude that strong shocks are unlikely and components of different optical depths share a common velocity field.

\subsection{Estimates of Ionizing Luminosity}

We can test for nuclear photoionization by comparing the UV ionizing flux of the AGN with the total emission line luminosity. Since the direct UV flux of the AGN is obscured, we use the FIR luminosity as a surrogate for the dust-reprocessed UV luminosity. From IRAS FSC measurements and estimates of geometrical and covering factors [see Whittle et al. (2004)a], we derive $L_{U V} \sim L_{F I R} \sim 10^{43.5} \mathrm{erg} \mathrm{s}^{-1}$, which is approximately equal to the total emission line luminosity, taken to be about $10 \times L_{5007}$. Clearly, an ionizing field from the AGN is sufficient to power the observed line emission in Mkn 78 and there is no need for any additional source of ionizing photons, such as fast shocks.

To conclude, all the evidence suggests that the principal ionizing source of $\mathrm{Mkn} 78$, and possibly most Seyferts, is the central AGN, coupled with a realistic multi-component ionized gas distribution. The role of ionizing shocks is negligible. This is borne out in detail in the dynamical analysis of Whittle et al. (2004)b.

\section{References}

Allen, M. G. 2004, private communication

Binette, L., Wilson, A. S., \& Storchi-Bergmann, T. 1996, A\&A, 312, 365

Baldwin, J. A., Phillips, M. M., \& Terlevich, R. 1981, PASP, 93, 5

Davidson, K., \& Netzer, H. 1979, Rev. Mod. Phys. 51, 715

Dopita, M. A., \& Sutherland, R. S. 1996, ApJS, 102, 161

Ferland, G. J. 1996, Hazy, a Brief Introduction to Cloudy, University of Kentucky Department of Physics and Astronomy Internal Report

Robinson, T. G., Tadhunter, C. N., Axon, D. J., \& Robinson, A. 2000, MNRAS, 317, 922

Viegas-Aldrovandi, S. M., \& Contini, M. 1989, ApJ, 339, 689

Whittle, M. 1992, ApJ, 387, 109

Whittle, M., \& Wilson, A. S. 2004, AJ, 127, 606 (Paper I)

Whittle, M., Rosario, D. J., Silverman, J. D., Nelson, C. H., \& Wilson, A. S. 2004a, AJ, submitted (Paper II)

Whittle, M., Silverman, J. D., Rosario, D. J., Nelson, C. H., \& Wilson, A. S. 2004b, AJ, submitted (Paper III) 